\title{
REVIEW
}

\section{Diffusion/Perfusion MR Imaging of the Liver: Practice, Challenges, and Future}

\author{
Masayuki Kanematsu ${ }^{*}$, Satoshi Goshima ${ }^{1}$, Haruo Watanabe ${ }^{1}$, Hiroshi Kondo ${ }^{1}$, \\ Hiroshi KAWADA ${ }^{1}$, Yoshifumi NODA ${ }^{1}$, and Noriyuki MORIYAMA ${ }^{2}$ \\ ${ }^{1}$ Department of Radiology, Gifu University Hospital \\ 1-1 Yanagido, Gifu 501-1194, Japan \\ ${ }^{2}$ Research Center for Cancer Prevention and Screening, National Cancer Center Hospital, Tsukiji, Japan
}

(Received February 3, 2012; Accepted May 17, 2012)

\begin{abstract}
Diffusion-weighted (DWI) magnetic resonance (MR) imaging is useful in diagnosing various pathologic conditions in the liver, such as malignant tumors or hepatic fibrosis, and is now part of routine MR imaging protocols for the liver following the development of a parallel encoding technique that has markedly improved image quality. DWI is not very sensitive for detecting hepatocellular carcinomas (HCC) and is useless for characterizing border-line hepatocellular nodules in cirrhosis, but it complements gadolinium-enhanced MR imaging in detecting regional tumor recurrence or intrahepatic metastases of HCC following treatment. DWI is more useful for detecting hepatic metastasis, because histopathologic architecture of metastases does not resemble that of liver tissue and $\mathrm{T}_{2}$ relaxation time of hepatic metasitasis is fairly longer than that of liver parenchyma. DWI is also useful for detecting moderate and advanced hepatic fibrosis. In cirrhosis, however, decreased blood flow in fibrotic liver is thought to lower apparent diffusion coefficient of the liver.

For MR perfusion analysis, a dual-input one-compartment model is used to correlate various hepatic blood flow parameters that represent hepatic arterial/portal blood flow or fraction, mean transit time, and distribution volume with the severity of cirrhosis and portal hypertension. Conventional multisectional imaging and perfusion study can be combined using a 3-dimensional sequence with high temporal resolution, but spatial resolution is not sufficiently high to diagnose tiny hepatic lesions. The advent of liver-specific contrast agents, such as gadoxetic acid, may spur the development of a new analysis model that incorporates extracellular perfusion and hepatocyte function.
\end{abstract}

Keywords: diffusion, prefusion, magnetic resonance imaging, liver

\section{Diffusion-weighted MR Imaging of the Liver}

An epoch-making application of diffusionweighted (DWI) magnetic resonance (MR) imaging for visualizing the restriction of water molecule diffusion in the hyperacute diagnosis of brain infarction was introduced in 1986. DWI sensitively visualized the area of cytotoxic edema caused by acute ischemia and subsequent insufficiency of the sodium-potassium pump activated by an adenosine triphosphoric acid cycle. ${ }^{1}$ Thereafter, DWI was shown to be useful in assessing a variety of pathologic conditions, including malignant tumors with hypercellularity that narrowed intercellular spaces and increased density of cell membranes to hamper

*Corresponding author, Phone: + 81-58-230-6439, Fax: + 8158-230-6440,E-mail: masa_gif@yahoo.co.jp diffusion of water molecules ${ }^{2}$ (Fig. 1); abscesses containing viscous pus consisting of inflammatory cells, bacteria, necrotic tissue, and proteinaceous exudates $^{3}$; and hemorrhage/hematoma with progressive thrombosis and solidly conglomerated red blood cells preceding erythrocytolysis and scarce plasma. ${ }^{4}$ All these disease conditions, which also occur in the liver, are evident as areas of abnormal intensity or decreased apparent diffusion coefficient (ADC) with DW imaging and require differentiation.

The advent of parallel encoding techniques has markedly improved the quality of DW images, which previously suffered from artifacts, such as ghosting or distortion caused by respiratory/cardiac motion or neighboring pulmonary/intestinal air. As a result, DWI has been adopted by many medical centers for MR imaging of the liver, ${ }^{5}$ is now part 


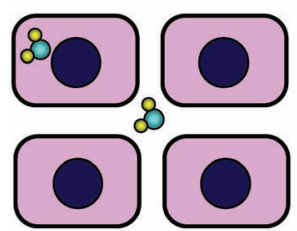

Normal tissue Benign tumor

Fig. 1. Schema showing water molecular diffusion properties in normal tissue or benign tumors (left) and malignant tumors (right). Intra- and extracellular molecular diffusion is intact in isocellular condition in normal or benign neoplastic tissue, but extracellular diffusion is restricted in hypercellular condition caused by malignant neoplasm.

of routine MR imaging protocols for detecting and characterizing focal hepatic lesions, and has served as an important supplementary sequence for $\mathrm{T}_{1^{-}} / \mathrm{T}_{2^{-}}$ weighted and gadolinium-enhanced MR imaging. However, the quality of DW images is readily degraded by physiological motion and internal air and depends on machine performance, and such degradation often leads to false positive/negative findings. It remains unclear whether DWI can be a primary imaging sequence and obviate the need for contrast-enhanced MR imaging.

Imaging techniques and parameters for DWI of the liver vary from institution to institution. Choice of acquisition using free-breathing, respiratorytriggering, navigator-tracking, or breath-hold is optional, but this selection influences image quality, signal-to-noise ratio (SNR), artifacts, and acquisition time. ${ }^{6}$ The $b$ value is an integrated parameter that regulates the strength, duration, and interval of bipolar motion-probing gradients in a DW sequence and affects the degree of phase dispersion and the diffusion weighting of images. Though moderate $b$ values around $500 \mathrm{~s} / \mathrm{mm}^{2}$ may be preferred for liver imaging to ensure image quality, differentiation between malignant and benign lesions is still challenging even with moderate $b$ values.

\section{DW imaging for hepatocellular nodules in cirrhosis}

Various kinds of hepatocellular nodules develop in the cirrhotic liver and include large regenerative nodules, low or high grade dysplasia, early hepatocellular carcinoma (HCC), and progressed HCC. ${ }^{7,8}$ $\mathrm{HCC}$ is the most common primary liver cancer and generally develops secondarily to chronic hepatitis or cirrhosis caused by viral hepatitis, alcoholism, autoimmune hepatitis, or non-alcoholic steatohepatitis. The diagnostic accuracy of MR imaging for HCCs has dramatically improved owing to the development of high performance MR imagers and gadolinium chelate contrast media. Hepatic nodules in cirrhosis as small as 10 to $15 \mathrm{~mm}$ in diameter are often detected on MR images and challenge radiologists to differentiate them among dysplasia, early HCC, and progressed HCC. Histopathologically, dysplasia and early HCC are differentiated based on the presence of stromal invasion. ${ }^{8}$ These border-line lesions are often revealed on ultrasonography or MR imaging, but most are iso- or hypovascular to the liver and cannot be differentiated by dynamic contrast-enhanced MR imaging with extracellular contrast material. Recently, however, a liver-specific contrast material, gadoxetic acid (Primovist $^{\mathrm{TM}} /$ Eovist $^{\mathrm{R}}$, Bayer Healthcare, Erlangen, Germany), was reported useful for differentiating dysplasia and early HCC. ${ }^{9}$

Detection of HCCs with DW imaging has been reported as low as $50 \%$ in evaluation using explanted livers (Fig. 2) ${ }^{10}$; DWI is virtually useless in detecting and characterizing border-line lesions in cirrhosis, such as high grade dysplasia and early HCC. DWI demonstration of HCCs that have acquired hepatic arterial vascularity may be explained by the increased cellularity or prolonged $T_{2}$ relaxation time in the hypervascular HCCs. Nevertheless, ADC measurement with DWI may help estimate the tumor grade of these HCCs. ADCs tend to decrease in the order of well, moderately, and poorly differentiated HCCs, but it may be unrealistic to predict tumor grade according to ADC value because of the large overlap of ADCs among histologic grades. ${ }^{11,12}$ Still, an ADC value exceeding $0.9 \times 10^{-3} \mathrm{~mm}^{2} / \mathrm{s}$ may reflect the low possibility of poorly differentiated HCC that could predispose patients to post-therapeutic tumor recurrence and poor prognosis. ${ }^{12}$

Therapeutic options for HCCs include liver transplantation, partial hepatectomy, transcatheter arterial chemoembolization (TACE), thermal ablation, and irradiation. Intrahepatic metastases or post-therapeutic recurrence of $\mathrm{HCC}$ often involves moderately or poorly differentiated $\mathrm{HCC},{ }^{13}$ and DWI can complement gadolinium-enhanced MR imaging to detect regional recurrence or intrahepatic metastasis of $\mathrm{HCC}$ (Fig. 3). ${ }^{14}$ After invasive treatments, early enhancing pseudolesions may develop in the liver due to arterioportal shunting and hamper the correct diagnosis of recurrence of hypervascular HCCs, but DW imaging helps detect hypervascular HCCs without interference from vascular pseudolesions. ${ }^{15}$ 
a
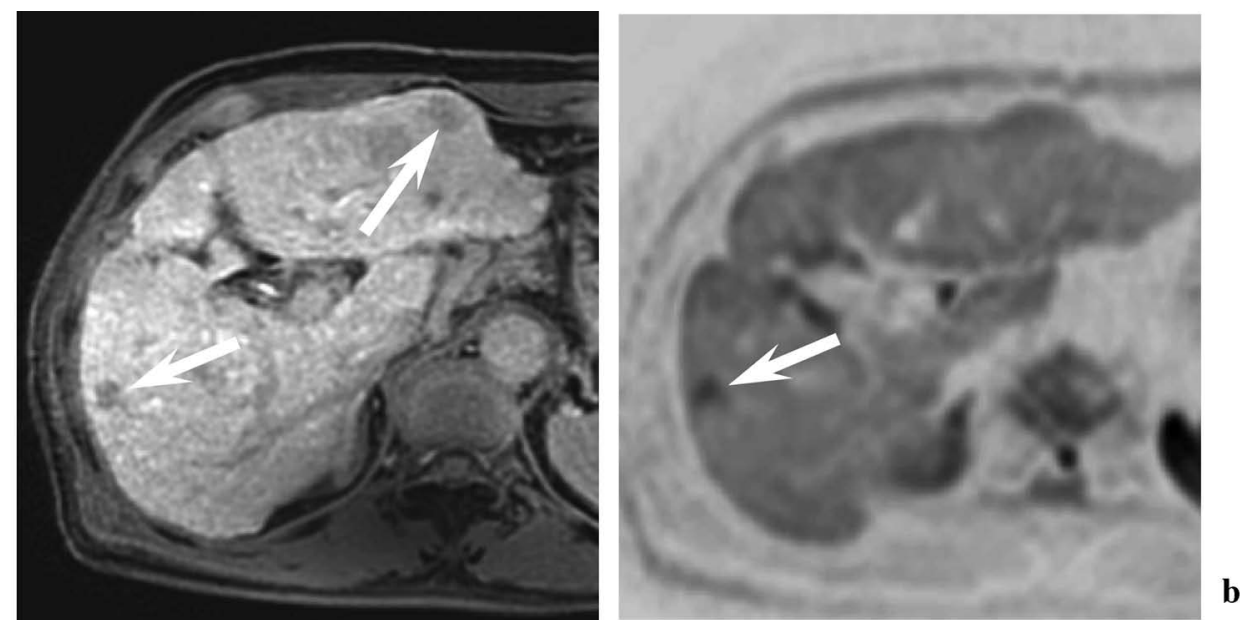

Fig. 2. Gadoxetic acid-enhanced hepatocyte-phase fat-suppressed $T_{1}$-weighted 3-dimensional gradient-echo axial (a) and diffusion-weighted (DW) ( $b$ value, 500 $\left.\mathrm{s} / \mathrm{mm}^{2}\right)$ (b) images obtained in a patient with type $\mathrm{C}$ hepatitis-related cirrhosis and hepatocellular carcinomas (HCCs) in segments III and VIII (arrows in a). Note that HCC in segment VIII of the liver is shown as area of increased signal intensity (arrow in b) on DW image, but HCC in segment III is not evidently exhibited.

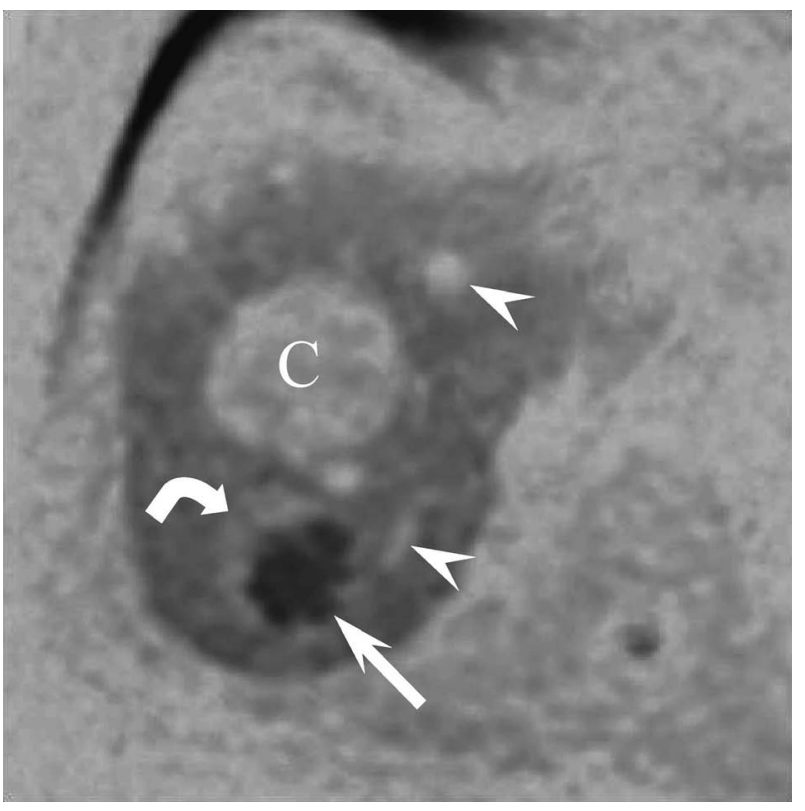

Fig. 3. Diffusion-weighted (DW) image ( $b$ value, $800 \mathrm{~s} / \mathrm{mm}^{2}$ ) in a patient with hepatocellular carcinoma (HCC) patient following transcatheter arterial chemoembolization (TACE). Residual HCC is shown as an area of increased signal intensity (arrow) in the vicinity of an area of coagulative necrosis exhibiting decreased signal intensity (curved arrow). Note that the hepatic cyst (C) and portal and hepatic veins (arrowheads) show areas in which there is no signal.

Occasionally, DWI is not as reliable as gadolinium-enhanced MR imaging for detecting local recur- rence of HCC after TACE. ${ }^{16}$ Coagulative or liquefactive necrosis can occur following TACE for HCC. ${ }^{17}$ Although coagulative necrosis may not be manifested as hyperintensity of DW images due to the paucity of water molecules, per se, liquefactive necrosis resulting from cellular membrane lysis following embolization may show $\mathrm{T}_{2}$ shine-through effect due to the prolonged regional $T_{2}$ relaxation time, and such a pseudolesion should be differentiated by reference to ADC maps. ${ }^{16}$ Nevertheless, the high contrast resolution of DW imaging can be an outstanding means of alerting the clinician to lymph node or bone metastasis or to tumor seeding by preceding percutaneous procedures.

\section{DW imaging for hepatic metastases}

DW imaging is more useful for detecting hepatic metastases than HCCs, primarily because $\mathrm{T}_{2}$ relaxation time is uesally fairly long with most metastases and the histopathologic architecture of metastases does not resemble that of the surrounding liver parenchyma; secondarily, hepatic metastases usually develop in noncirrhotic livers without heterogeneous signal intensity from such causes as morphological change, multiple nodularity, fibrosis, necrosis, and iron deposition. Thus, the quality of DW images of the liver is stable with noncirrhotic livers (Fig. 4). ADC values of metastases are as low as $1.00 \times 10^{-3} \mathrm{~mm}^{2} / \mathrm{s}$ at $b$ values of 400 to 800 $\mathrm{s} / \mathrm{mm}^{2} .{ }^{18}$ One report described DWI as a reasonable alternative to gadolinium-enhanced dynamic MR imaging for detecting liver metastases ${ }^{19}$; an- 
a
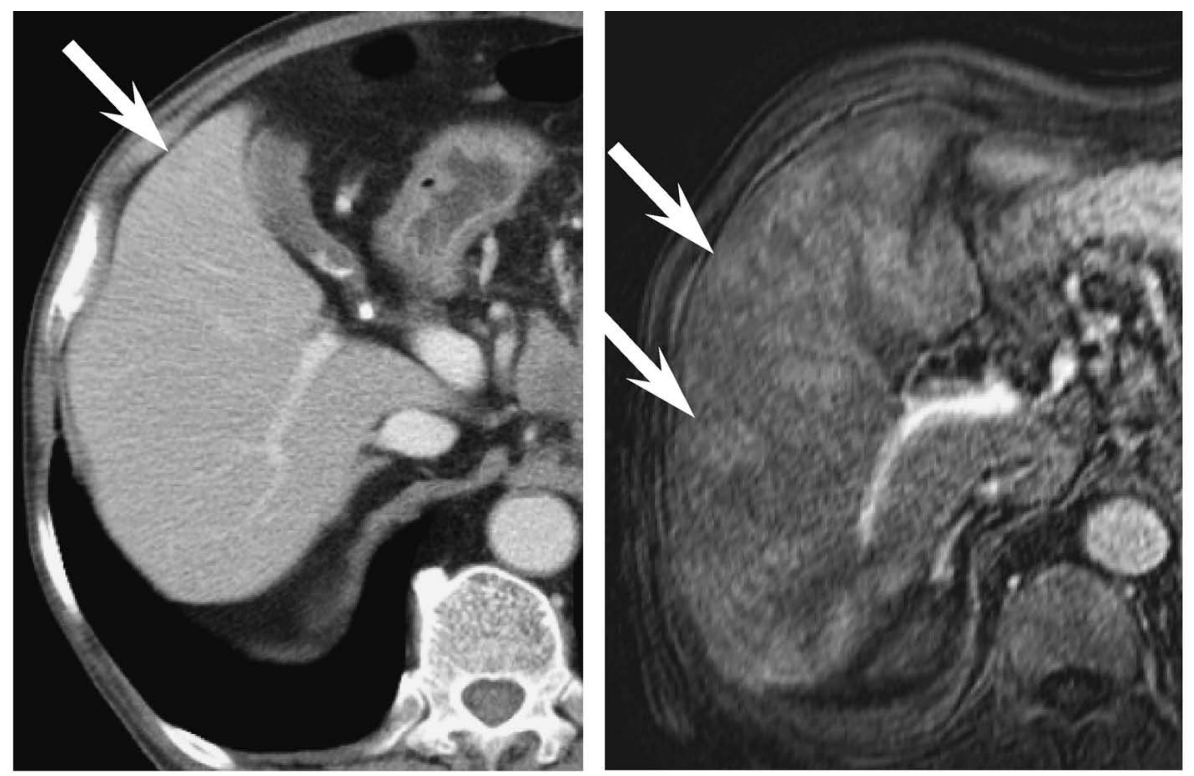

b

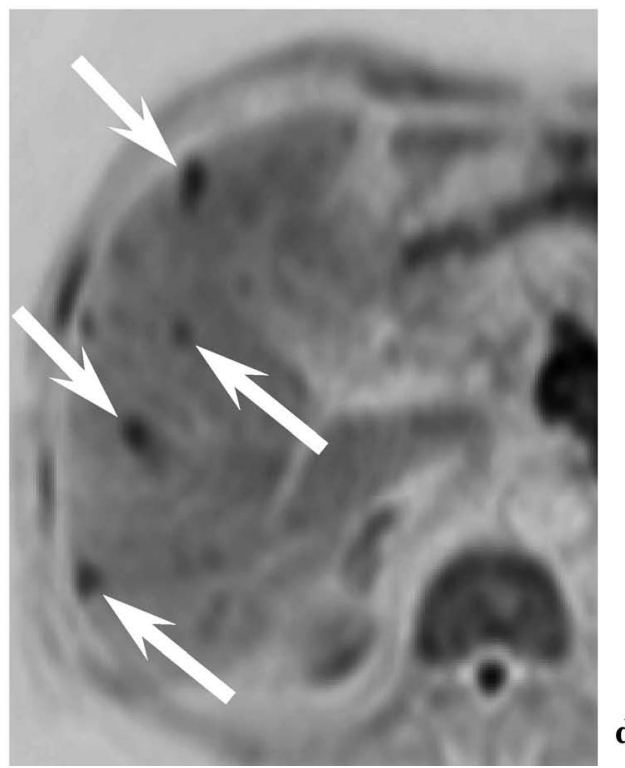

c

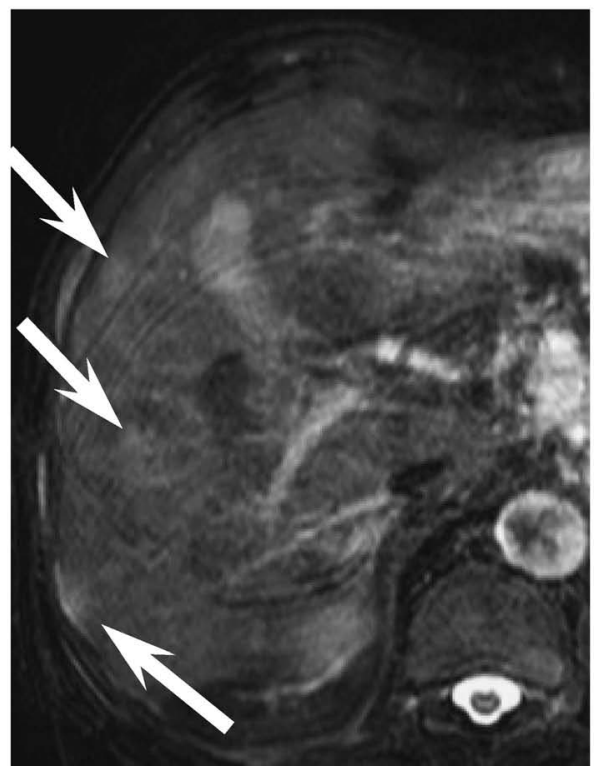

Fig. 4. Contrast-enhanced portal venous-phase computed tomography (CT) (a), gadolinium-enhanced hepatic arterial dominant-phase 3-dimensional gradient-echo (b), respiratory-triggered fat-suppressed $\mathrm{T}_{2}$-weighted fast spinecho (c), and diffusion-weighted (DW) ( $b$ value, $500 \mathrm{~s} / \mathrm{mm}_{2}$ ) (d) magnetic resonance (MR) images in a patient with advanced pancreatic cancer. Only one tiny slightly hypodense nodule (arrow in a) is noted on CT. Multiple areas of illdefined enhancement (arrows in b) are seen on gadolinium-enhanced image, but presence of metastases is indeterminate. Multiple areas of ill-defined, slight hyperintensity (arrows in c) suggesting the possibility of metastases are seen on $\mathrm{T}_{2}$-weighted image. Multiple areas of distinctly increased signal intensity (arrows in d) strongly suspicious of metastases are seen on DW image.

other demonstrated the superior accuracy of combining DW images with dual-echo $\mathrm{T}_{1}$-weighted and $\mathrm{T}_{2}$-weighted images to that using superparamagnetic iron oxide-enhanced MR images for detecting hepatic metastases. ${ }^{20}$ More recent reports have indicated that DWI may not be a suitable alternative to gadoxetic acid-enhanced MR imaging for detecting metastases ${ }^{21}$ and that better detection of small colorectal metastases may result from combining gadoxetic acid-enhanced MR imaging and DWI rather than using DWI alone. ${ }^{22}$

\section{Differentiating focal hepatic lesions with DW imag- ing}

ADC measurement with DW imaging is useful for differentiating focal hepatic lesions. Hepatic 
cysts are efficiently discriminated from HCCs, metastases, and cavernous hemangiomas with a cutoff ADC of $2.5 \times 10^{-3} \mathrm{~mm}^{2} / \mathrm{s}$ at $b$ values of 400 to $1,000 \mathrm{~s} / \mathrm{mm}^{2}$. However, though ADCs tend to decrease in the order of hemangiomas, HCCs, and metastases, the substantial overlap of ADC values among these pathologies makes differentiation of the conditions virtually impossible using any $b$ value. ${ }^{18}$

Intravoxel incoherent motion (IVIM) imaging is an MR imaging method that enables separate determination of coefficients with molecular diffusion of water and microcirculation of blood in the capillary network in each imaging voxel. These 2 phenomena account for the biexponential decay of signal observed on DWI with the application of different diffusion $b$ values. The IVIM sequence allows extraction of 2 diffusion coefficients-one related to restriction of molecular diffusion (D) and another related to tissue perfusion $\left(\mathrm{D}^{*}\right)$ - and finally the vascular volume fraction $(f)$ (Fig. 5). ${ }^{23}$ The utility of the values of $\mathrm{D}$ and $f$ has been described for characterizing hepatic lesions despite significant overlap of the 2 values among HCCs, metastases, and hemangiomas (Fig. 6). ${ }^{24}$

\section{Staging hepatic fibrosis with DW imaging}

Hepatic fibrosis is an irreversible pathologic condition that causes hardening of the liver, impairs hepatic functional reserve after global hepatocyte necrosis, and subsequent proliferation of collagenous fibers when there is long-standing chronic liver damage caused by such factors as viral hepatitis, alcohol abuse, autoimmune hepatitis, and nonalcoholic steatohepatitis. The degree of hepatic fibrosis is closely related to hepatic functional reserve or prognosis. Because the risk for developing HCC increases with the degree of hepatic fibrosis, ${ }^{25}$ and severe fibrosis (F3) or cirrhosis (F4) is associated with decreased response to antiviral therapy in chronic hepatitis $\mathrm{C},{ }^{26}$ the discrimination of hepatic fibrosis stages $\leq \mathrm{F} 2$ and $\geq \mathrm{F} 3$ may be crucial in considering the indication and efficacy of antiviral therapies. Although biopsy is currently a standard-of-reference method for grading fibrosis, it is invasive, only locally diagnostic, and not necessarily accurate. Laboratory tests, such as aspartate transaminase-to-platelet ratio index (APRI), FibroTest, and Forns index are useful, but MR imaging has been and remains the promising modality of choice for evaluating hepatic fibrosis. Conventional unenhanced $\mathrm{T}_{1} / \mathrm{T}_{2}$-weighted ${ }^{27}$ and gadolinium-enhanced ${ }^{28}$ MR images are still useful in the evaluation of morphological, volumetric, and textural changes of the liver that occur with cir- rhosis.

Several studies have shown that ADCs are lower for the cirrhotic liver than normal liver, possibly because of the greater presence of connective tissue in the liver, narrowed sinusoids, and decreased blood flow with cirrhosis. ${ }^{29} \mathrm{DW}$ imaging can be used for assessing the presence of moderate and advanced liver fibrosis. In one study, the sensitivity for hepatic fibrosis of $\mathrm{F} 3$ or $\mathrm{F} 4$ was $70 \%$ and specificity, $85 \%$, at a $b$ value of $500 \mathrm{~s} / \mathrm{mm}^{2}$, whereas sensitivity was as high as $80 \%$ and specificity, $90 \%$, at $1,000 \mathrm{~s} / \mathrm{mm}^{2}{ }^{30}$ Though the use of high $b$ values seems advantageous for evaluating hepatic fibrosis, associated tradeoffs include blackout, distortion, or ill-defined visceral contours affected by low SNR, physiological motion (cardiac, respiratory, intestinal), and internal air. Accordingly, many institutions prefer using moderate $b$ values around 500 $\mathrm{s} / \mathrm{mm}^{2}$ for DW imaging of the liver.

Correlation of pure molecular-based (D) and perfusion-related $\left(\mathrm{D}^{*}, f\right)$ diffusion parameters using an IVIM sequence showed no difference in D between patients with cirrhosis and without. Restricted diffusion observed in patients with cirrhosis may be related to variations in $\mathrm{D}^{*}$, which reflected decreased perfusion. Decreased ADC was chiefly caused by the decrease in total hepatic blood flow in cirrhosis. $^{31}$

\section{Perfusion MR Imaging of the Liver}

In the early 1990s, perfusion MR imaging was introduced as a technique for imaging regional and global blood flow of the heart ${ }^{32}$ and lung ${ }^{33}$ using a $\mathrm{T}_{1}$-weighted gradient-recalled-echo sequence and that of the brain ${ }^{34}$ using a susceptibility-weighted sequence. Myocardial perfusion MR imaging detected functionally significant coronary heart disease with excellent diagnostic performance. ${ }^{35}$ Diffusion/perfusion MR imaging of the brain demonstrated the prowess of the approach in the detection of ischemic penumbra to be salvaged by preemptive treatments. ${ }^{36} \mathrm{MR}$ perfusion study has also been applied to bone marrow, kidney, and prostate to evaluate blood flow, function, and neoplasm.

A report on MR perfusion study of the liver using gadolinium in rats was published in $1994^{37}$ and followed by reports of studies using superparamagnetic iron oxide ${ }^{38}$ and gadolinium ${ }^{39}$ in human subjects. Introduction of dual-input one-compartment analysis (Fig. 7) permitted measurement of such hepatic blood flow parameters as hepatic arterial/ portal venous inflow rate constant $\left(\mathrm{k}_{1 \mathrm{a}} / \mathrm{k}_{1 \mathrm{p}}\right)$, absolute hepatic arterial/portal venous blood flow $(\mathrm{mL} / 100 \mathrm{~g} / \mathrm{min})$, hepatic arterial/portal venous 


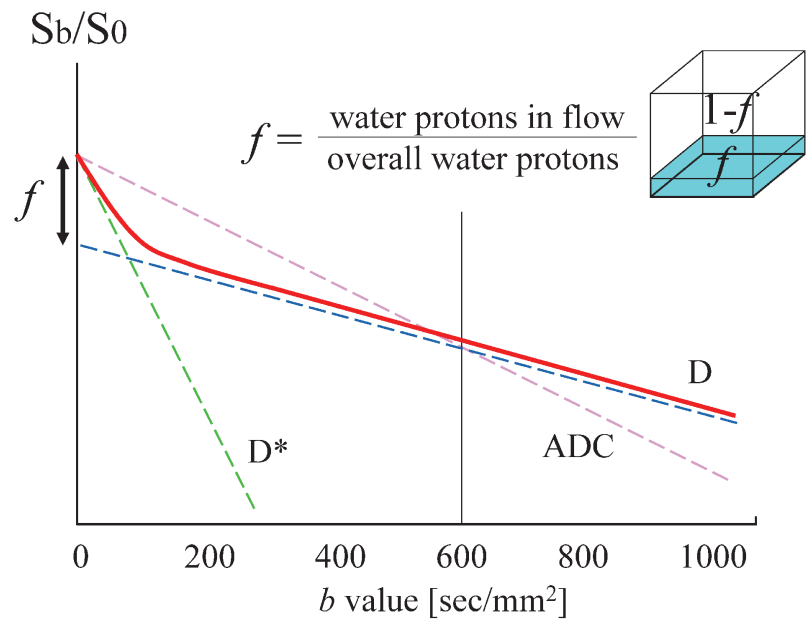

Fig. 5. Schema showing biexponential fitting curve (red solid line) and simple regression lines yielding true molecular diffusion coefficient, D (blue broken line), tissue microperfusion-related diffusion coefficient, $\mathrm{D}^{*}$, (green broken line), and apparent diffusion coefficient (pink broken line). Difference between $y$ intercepts with $\mathrm{D}$ and $\mathrm{D}^{*}$ is interpreted as $f$, which corresponds to the fraction of water protons in microperfusion to overall water protons.

$\mathbf{a}$
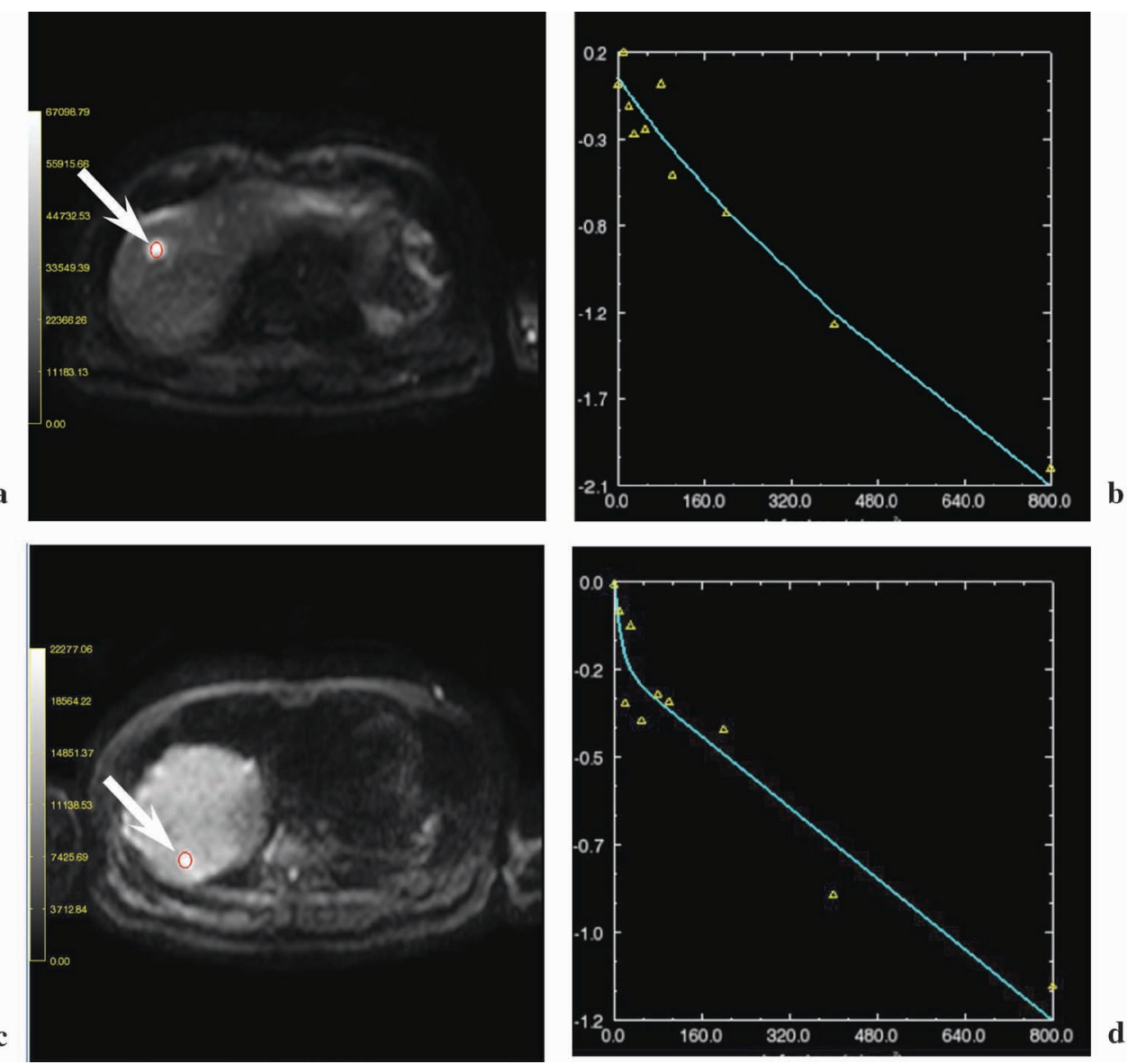

Fig. 6. Intravoxel incoherent motion (IVIM) diffusion-weighted (DW) image (a) and biexponential fitting curve (b) in a patient with hepatic cysts and those (c and $\mathbf{d}$, respectively) in a patient with hepatocellular carcinoma (HCC). Note the hepatic cyst with a circular region of interest (arrow in a) placed in segment VIII of the liver. The $\mathrm{D}$ of this cyst is 2.12 ; $\mathrm{D}^{*}$ is 5.00 ; and $f$ is 0.39 . The microperfusion fraction, $f$, is paradoxically high in this specific case, which is presumed to be a measurement error. Note the HCC with a circular region of interest (arrow in c) placed in segment VII of the liver. The D of this HCC is 1.27 ; $\mathrm{D}^{*}$ is 78.30 ; and $f$ is 0.21 . $\mathrm{D}^{*}$ is very high probably because the flow-related water molecule diffusion is vigorous in this hypervascular HCC. 


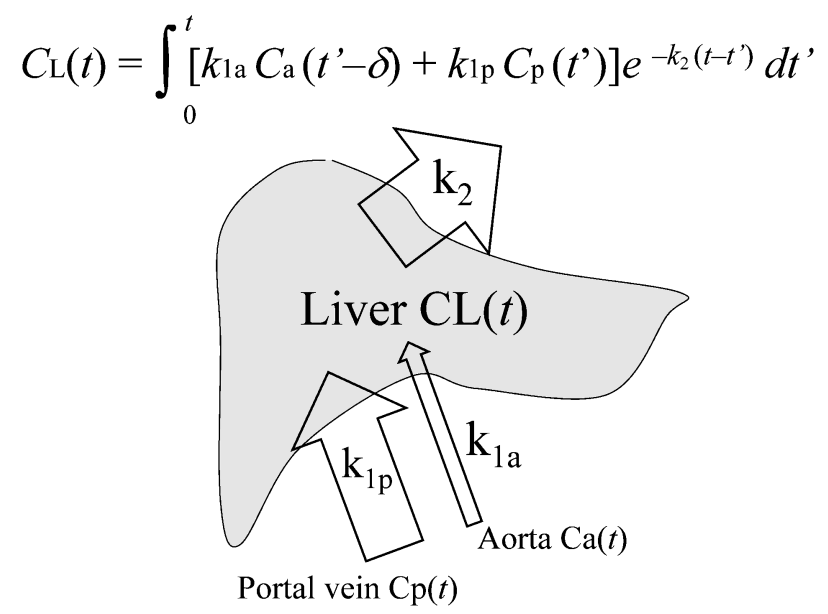

Fig. 7. Function formula and schema showing theory of dual-input one-compartment analysis with magnetic resonance (MR) perfusion study of the liver. Introduction of dual-input one-compartment analysis allows determination of hepatic arterial $\left(\mathrm{k}_{1 \mathrm{a}}\right)$ and portal venous $\left(\mathrm{k}_{1 \mathrm{p}}\right)$ inflow rate constants, absolute hepatic arterial and portal venous blood flows (mL/100 g/min), hepatic arterial and portal venous fractions (\%), mean transit time (s), and distribution volume $(\%)$.

fraction (\%), mean transit time (s), and distribution volume (\%) with MR perfusion imaging and correlation with severity of cirrhosis and portal hypertension. ${ }^{39}$ Nevertheless, for the evaluation of hepatic fibrosis, functional reserve, and neoplasm, MR perfusion imaging is not established as an alternative to other clinical tests, such as serological, sonographic, and conventional MR and histopathologic examinations, in terms of accuracy, noninvasiveness, and cost.

Perfusion MR imaging is intended to assess regional and global changes in the distribution of blood flow. Because of the unique incoming blood flow in the liver via dual pathways - the hepatic artery and portal vein-hepatic arterial and portal venous fractions as well as total blood flow are important perfusion parameters for assessing chronic liver disease characterized as hepatic fibrosis or cirrhosis. Breedis and Young first described the primary, if not exclusive, arterial blood supply of hepatic tumors in contrast to the predominantly portal supply of hepatic tissue. ${ }^{40}$ Portal venous perfusion may be disregarded in evaluating perfusion properties in focal hepatic lesions, and first-pass perfusion analysis has been used successfully to evaluate tumor vascularity in advanced HCC. ${ }^{41}$

In normal liver, normal fenestrae between the sinusoidal endothelial cells allow free passage of blood plasma and gadolinium chelate molecules be- tween hepatic sinusoids and the space of Disse. In cirrhosis, a narrowing or loss of fenestrae associated with deposition of collagenous fibers in the space of Disse causes sinusoids to become more capillary-like, impairs the transfer of plasma and low molecular-weight compounds, such as gadolinium chelate, from the sinusoids into the space of Disse, and increases vascular resistance (Fig. 8). Such changes in circulatory dynamics caused by reduction of the vascular bed of the liver are reflected in decreased portal flow in advanced cirrhosis as determined from MR perfusion analyses.

For perfusion MR imaging of the liver to be feasible, a tracer that accurately determines concentration, an imaging method with spatial and temporary resolutions that permit generation of perfusion curves, and a robust pharmacokinetic model that gives blood flow, blood volume, mean transit time, and arterial fraction are necessary. More ideally, for MR perfusion study as a one-stop examination, an imaging method covering the whole liver, capability of quantitative determination of total, segmental, and lesional blood flow, and high spatial resolution sufficient for detecting and characterizing small hepatic lesions would be required.

Initially, MR perfusion studies were performed as continuous imaging of a single transverse section passing through the portal vein, aorta, and right hepatic lobe with a $T_{1}$-weighted fast spoiled gradient-echo sequence. This enabled temporal resolution as short as one second and perfusion curve fitting with dual-input one-compartment analysis. ${ }^{39}$ Later, 3-dimensional (3D) perfusion MR imaging that covered the entire liver was developed to evaluate global and regional changes in perfusion in the liver, which could be useful in diagnosing fibrosis, detecting interval changes during antiviral treatment monitoring, and screening for HCC. ${ }^{42}$ In this study, 36 to 40 coronal images were acquired every 3.3 to $5.0 \mathrm{~s}$ for approximately $2 \mathrm{~min}$ after contrast administration, and the images were successfully fit to perfusion curves with dual-input one-compartment analysis to output hepatic arterial/portal/ total blood flow, hepatic arterial/portal venous fraction, mean transit time, and distribution volume. ${ }^{42}$ Previous studies evaluating chronic liver damage from hepatic fibrosis demonstrated increases of blood flow/fraction of the hepatic artery, mean transit time, and distribution volume, while decreases of total/portal blood flow and portal venous fraction, as the stage of hepatic fibrosis advanced (Fig. 9) ${ }^{39,42}$ Regretfully, the low spatial resolution of MR perfusion study using a 3D sequence with 256 by 128 matrices and coronal-plane 
imaging that comes as a tradeoff with its high temporal resolution to ensure perfusion curve fitting limits correlation with routine transaxial imaging and evaluation of tiny hepatic lesions. A future goal to establish the usefulness and role of MR per-

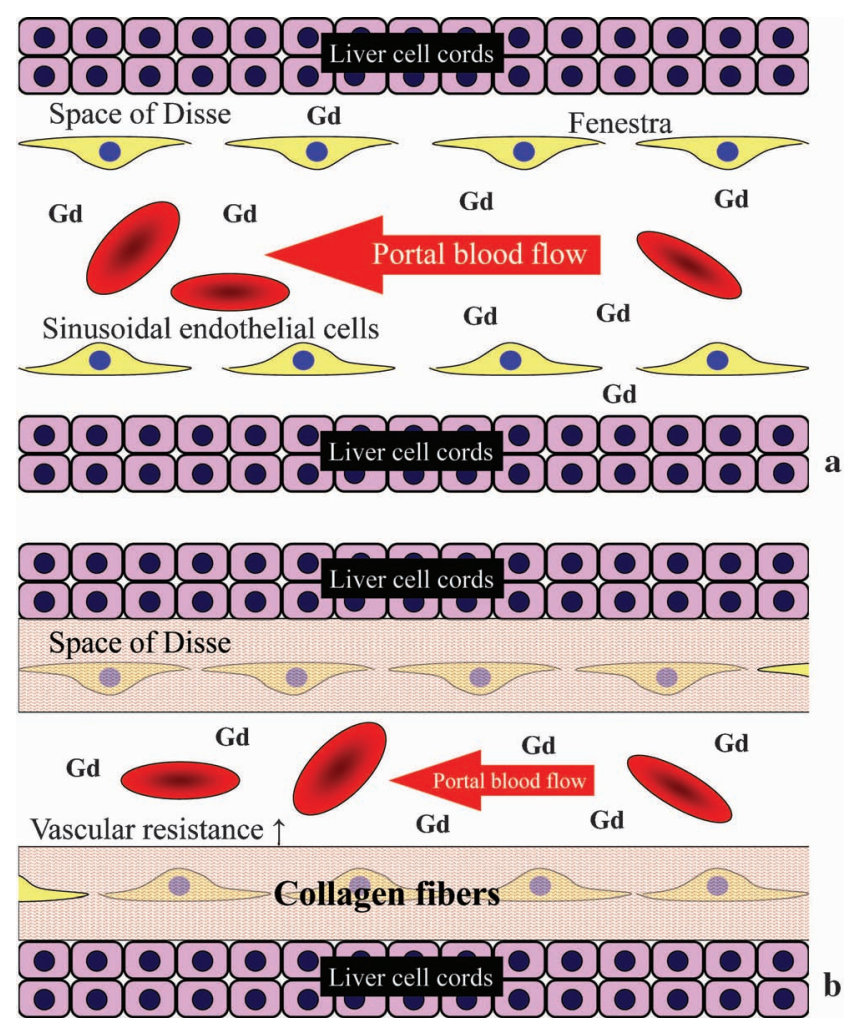

fusion study in radiology practice will be the development of high spatial resolution imaging that permits evaluation of tiny focal hepatic lesions and high temporal resolution imaging that allow perfusion analysis in a single session (Fig. 10).

More recently, gadoxetic acid has been widely used in evaluating extracellular perfusion and hepatocyte functional properties with focal hepatic lesions ${ }^{43}$ and the liver. ${ }^{44}$ In Japan, Conventional gadolinium-chelate contrast material used for MR perfusion studies is barely used in routine MR imaging of the liver, in which the main purpose is the detection of malignant hepatic tumors. Gadoxetic acid is taken up by hepatocytes early in the extracellular phase and exhibits a unique time-signal

Fig. 8. Schema showing pathophysiological and hemodynamics differences between normal (a) and cirrhotic (b) liver. In normal liver (a), the normal fenestrae between sinusoidal endothelial cells allow free passage of blood plasma and gadolinium chelate molecules between hepatic sinusoids and the space of Disse. In cirrhosis (b), narrowing or loss of fenestrae associated with deposition of collagenous fibers in the space of Disse causes sinusoids to become more capillary-like, which impairs transfer of plasma and low molecular-weight compounds, such as gadolinium chelate, from sinusoids into the space of Disse and leads to increased vascular resistance.

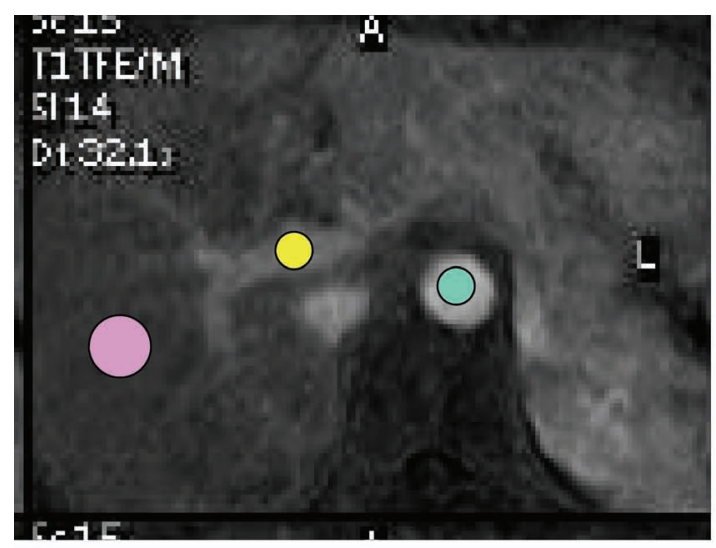

a

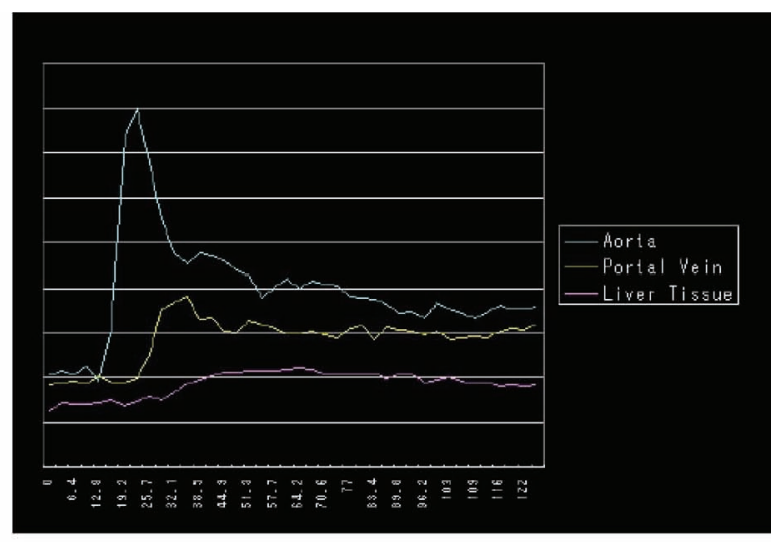

b

Fig. 10. An example case of split-dose magnetic resonance (MR) perfusion study. Image showing a representative portal inflow-phase image of serial single-level MR perfusion imaging with intravenous bolus injection of $5 \mathrm{~mL}$ of gadolinium-chelate contrast (a). The split-dose MR perfusion study was conducted following acquisition of routine gadolinium-enhanced dynamic MR imaging with $10 \mathrm{~mL}$ of gadolinium contrast. Graph showing the time-intensity curves for the abdominal aorta, portal vein, and liver parenchyma (b). Note colored circles in a showing typical locations of regions of interest for measurement of signal intensity in the abdominal aorta (blue), portal vein (yellow), and liver parenchyma (pink). In this patient, the hepatic arterial flow was determined as $21.2 \mathrm{~mL} / 100 \mathrm{~g} / \mathrm{min}$, the portal venous flow, as $99.3 \mathrm{~mL} / 100 \mathrm{~g} / \mathrm{min}$, and the total hepatic flow as $120.5 \mathrm{~mL} / 100 \mathrm{~g} / \mathrm{min}$. 


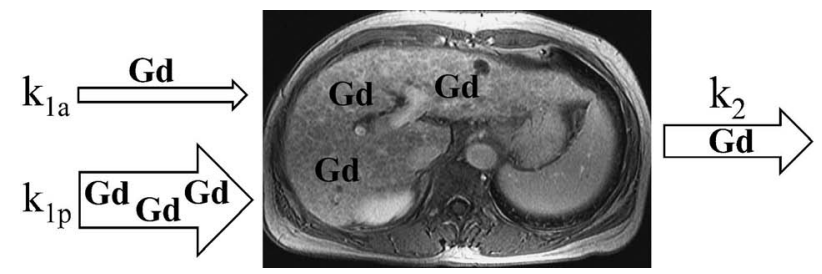

Fig. 9. Schema showing mechanism of increase of mean transit time $\left(\mathrm{MTT}=1 / \mathrm{k}_{2}\right)$ and distribution volume $\left(\mathrm{DV}=\left[\mathrm{k}_{1 \mathrm{a}}+\mathrm{k}_{1 \mathrm{p}}\right] / \mathrm{k}_{2} \times 100\right)$ with magnetic resonance (MR) perfusion study. As the interstitial area expands with advancing hepatic fibrosis, the transfer of more gadolinium chelate molecules into the interstitial spaces in liver tissue impair clearance of contrast material from the liver via hepatic veins. Temporary retention of more gadolinium contrast in liver tissue increases MTT and DV, which is also explained by decrease of $\mathrm{k}_{2}$.

intensity profile that cannot be explained solely by extracellular space circulation. As a result, the conventional dual-input one-compartment model is no longer applicable, and a new analysis model that incorporates hepatic perfusion and hepatocyte function may be necessary.

\section{Molecular-targeting Therapy and Diffusion/ Perfusion MR Imaging}

Sorafenib is a small molecular inhibitor of several tyrosine protein kinases, such as vascular endothelial growth factor receptor (VEGFR) and platelet-derived growth factor (PDGFR), and Raf kinases, such as C-Raf and B-Raf. ${ }^{45}$ This agent is used to treat hepatocellular, renal cell, and breast carcinomas. One study reported characteristic but unusual changes in the ADC of HCCs during sorafenib therapy that included an early decrease, typically in 3 weeks, followed by an increase in 2 months and another decrease thereafter. The results suggested that the first decrease in ADC most likely reflected hemorrhagic necrosis induced by sorafenib, the subsequent increase reflected tumor necrosis, and the final decrease reflected tumor reactivation in the later follow-up period. ${ }^{46}$

Another study determined ADC, D, and $f$ with IVIM MR imaging in advanced HCCs during sorafenib treatment and found $f$ to be a valuable marker of sorafenib treatment for HCCs. ${ }^{47}$ The authors conjectured that the mechanism of increase of $f$ at 2 weeks in responder patients was an antiangiogenic effect of sorafenib. Furthermore, in addition to its destructive action on small, leaky, and poorly efficient tumor capillaries, sorafenib improved the quality of remaining vessels in terms of basement membrane thickness and pericyte coverage, resulting in decreased vascular permeability and interstitial fluid pressure. ${ }^{47}$

\section{Future Challenges with Diffusion/Perfusion MR Imaging of the Liver}

Diffusion-weighted sequences that are robust for arbitrary magnetic field strength, magnetic field inhomogeneity, patient cardiac and respiratory motion, susceptibility, or machine performance will be developed and more frequently applied to MR imaging of the liver. Refinement of breath-hold imaging techniques will improve patient throughput, and multi-transmit technology may dramatically improve the quality of DW images, especially at magnetic field strength of 3 tesla or greater. Pure diffusion properties of the liver and focal hepatic lesions will be evaluated more frequently using IVIM sequences. Further research of MR diffusion kurtosis $^{48}$ or contrast-enhanced MR imaging with chemical exchange saturation transfer (CEST) imaging 49 will also be attempted for the liver. For perfusion MR imaging, development of a more time-resolved sequence with high spatial resolution is urged to ensure the continuing efforts to enable one-stop MR imaging study that incorporates routine transectional imaging and perfusion analysis. Furthermore, considering the increasing utilization of gadoxetic acid, a robust analytic model for evaluating extracellular circulatory and hepatocyte functional properties will need to be developed. Within a decade, diffusion/perfusion MR imaging will play an important role in predicting and monitoring the efficacy of molecular-targeting therapy for hepatic tumors.

\section{Acknowledgement}

We received partial support from a Grant for Scientific Research Expenses for Health, Labor, and Welfare Programs, Foundation for the Promotion of Cancer Research, and Research on Cancer Prevention and Health Services.

\section{References}

1. Le Bihan D, Breton E, Lallemand D, Grenier P, Cabanis E, Laval-Jeantet M. MR imaging of intravoxel incoherent motions: application to diffusion and perfusion in neurologic disorders. Radiology 1986; 161:401-407.

2. Higano S, Yun X, Kumabe T, et al. Malignant astrocytic tumors: clinical importance of apparent diffusion coefficient in prediction of grade and 
prognosis. Radiology 2006; 241:839-846.

3. Kim YJ, Chang KH, Song IC, et al. Brain abscess and necrotic or cystic brain tumor: discrimination with signal intensity on diffusion-weighted MR imaging. AJR Am J Roentgenol 1998; 171:14871490.

4. Ebisu T, Tanaka C, Umeda M, et al. Hemorrhagic and nonhemorrhagic stroke: diagnosis with diffusion-weighted and $\mathrm{T}_{2}$-weighted echo-planar $\mathrm{MR}$ imaging. Radiology 1997; 203:823-828.

5. Sodickson DK. Tailored SMASH image reconstructions for robust in vivo parallel MR imaging. Magn Reson Med 2000; 44:243-251.

6. Taouli B, Sandberg A, Stemmer A, et al. Diffusion-weighted imaging of the liver: comparison of navigator triggered and breathhold acquisitions. J Magn Reson Imaging 2009; 30:561-568.

7. International Working Party. Terminology of nodular hepatocellular lesions. Hepatology 1995; 22:983-993.

8. International Consensus Group for Hepatocellular Neoplasia. Pathologic diagnosis of early hepatocellular carcinoma: a report of the international consensus group for hepatocellular neoplasia. Hepatology 2009; 49:658-664.

9. Sano K, Ichikawa T, Motosugi U, et al. Imaging study of early hepatocellular carcinoma: usefulness of gadoxetic acid-enhanced MR imaging. Radiology $2011 ; 261: 834-844$.

10. Hardie AD, Kizziah MK, Rissing MS. Can the patient with cirrhosis be imaged for hepatocellular carcinoma without gadolinium?: Comparison of combined $\mathrm{T}_{2}$-weighted, $\mathrm{T}_{2}{ }^{*}$-weighted, and diffusion-weighted MRI with gadolinium-enhanced MRI using liver explantation standard. J Comput Assist Tomogr 2011; 35:711-715.

11. Nasu K, Kuroki Y, Tsukamoto T, Nakajima H, Mori K, Minami M. Diffusion-weighted imaging of surgically resected hepatocellular carcinoma: imaging characteristics and relationship among signal intensity, apparent diffusion coefficient, and histopathologic grade. AJR Am J Roentgenol 2009; 193:438-444.

12. Nishie A, Tajima T, Asayama Y, et al. Diagnostic performance of apparent diffusion coefficient for predicting histological grade of hepatocellular carcinoma. Eur J Radiol 2011; 80:e29-e33.

13. Nakashima O, Kojiro M. Recurrence of hepatocellular carcinoma: multicentric occurrence or intrahepatic metastasis? A viewpoint in terms of pathology. J Hepatobiliary Pancreat Surg 2001; 8:404409.

14. Yu JS, Chung JJ, Kim JH, et al. Detection of small intrahepatic metastases of hepatocellular carcinomas using diffusion-weighted imaging: comparison with conventional dynamic MRI. Magn Reson Imaging 2011; 29:985-992.

15. Motosugi U, Ichikawa $\mathrm{T}$, Sou $\mathrm{H}$, et al. Distinguishing hypervascular pseudolesions of the liver from hypervascular hepatocellular carcinomas with gadoxetic acid-enhanced MR imaging. Radiology 2010; 256:151-158.

16. Goshima S, Kanematsu M, Kondo H, et al. Evaluating local hepatocellular carcinoma recurrence post-transcatheter arterial chemoembolization: is diffusion-weighted MRI reliable as an indicator? J Magn Reson Imaging 2008; 27:834-839.

17. Yoshioka H, Nakagawa K, Shindou H, et al. MR imaging of the liver before and after transcatheter hepatic chemo-embolization for hepatocellular carcinoma. Acta Radiol 1990; 31:63-67.

18. Goshima S, Kanematsu M, Kondo H, et al. Diffusion-weighted imaging of the liver: optimizing $b$ value for the detection and characterization of benign and malignant hepatic lesions. J Magn Reson Imaging 2008; 28:691-697.

19. Hardie AD, Naik M, Hecht EM, et al. Diagnosis of liver metastases: value of diffusion-weighted MRI compared with gadolinium-enhanced MRI. Eur Radiol 2010; 20:1431-1441.

20. Nasu K, Kuroki Y, Nawano S, et al. Hepatic metastases: diffusion-weighted sensitivity-encoding versus SPIO-enhanced MR imaging. Radiology 2006; 239:122-130.

21. Shimada $\mathrm{K}$, Isoda $\mathrm{H}$, Hirokawa $\mathrm{Y}$, Arizono $\mathrm{S}$, Shibata T, Togashi K. Comparison of gadoliniumEOB-DTPA-enhanced and diffusion-weighted liver MRI for detection of small hepatic metastases. Eur Radiol 2010; 20:2690-2698.

22. Chung WS, Kim MJ, Chung YE, et al. Comparison of gadoxetic acid-enhanced dynamic imaging and diffusion-weighted imaging for the preoperative evaluation of colorectal liver metastases. J Magn Reson Imaging 2011; 34:345-353.

23. Le Bihan D, Breton E, Lallemand D, Aubin ML, Vignaud J, Laval-Jeantet M. Separation of diffusion and perfusion in intravoxel incoherent motion MR imaging. Radiology 1988; 168:497-505.

24. Yamada I, Aung W, Himeno Y, Nakagawa T, Shibuya H. Diffusion coefficients in abdominal organs and hepatic lesions: evaluation with intravoxel incoherent motion echo-planar MR imaging. Radiology 1999; 210:617-623.

25. Ikeda K, Saitoh S, Suzuki Y, et al. Disease progression and hepatocellular carcinogenesis in patients with chronic viral hepatitis: a prospective observation of 2215 patients. J Hepatol 1998; 28:930-938.

26. Lee SS. Review article: indicators and predictors of response to anti-viral therapy in chronic hepatitis C. Aliment Pharmacol Ther 2003; 17:611-621.

27. Ito K, Mitchell DG, Hann HW, et al. Progressive viral-induced cirrhosis: serial MR imaging findings and clinical correlation. Radiology 1998; 207:729735 .

28. Kato H, Kanematsu M, Zhang X, et al. Computeraided diagnosis of hepatic fibrosis: preliminary evaluation of MRI texture analysis using the finite difference method and an artificial neural network. 
AJR Am J Roentgenol 2007; 189:117-122.

29. Annet L, Peeters F, Abarca-Quinones J, Leclercq I, Moulin P, Van Beers BE. Assessment of diffusion-weighted MR imaging in liver fibrosis. J Magn Reson Imaging 2007; 25:122-128.

30. Taouli B, Tolia AJ, Losada M, et al. Diffusionweighted MRI for quantification of liver fibrosis: preliminary experience. AJR Am J Roentgenol 2007; 189:799-806.

31. Luciani A, Vignaud A, Cavet M, et al. Liver cirrhosis: intravoxel incoherent motion MR imaging--pilot study. Radiology 2008; 249:891-899.

32. Atkinson DJ, Burstein D, Edelman RR. First-pass cardiac perfusion: evaluation with ultrafast MR imaging. Radiology 1990; 174:757-762.

33. Berthezène $Y$, Vexler $\mathrm{V}$, Clément $\mathrm{O}$, Mühler $\mathrm{A}$, Moseley ME, Brasch RC. Contrast-enhanced MR imaging of the lung: assessments of ventilation and perfusion. Radiology 1992; 183:667-672.

34. Warach S, Levin JM, Schomer DL, Holman BL, Edelman RR. Hyperperfusion of ictal seizure focus demonstrated by MR perfusion imaging. AJNR Am J Neuroradiol 1994; 15:965-968.

35. Watkins S, McGeoch R, Lyne J, et al. Validation of magnetic resonance myocardial perfusion imaging with fractional flow reserve for the detection of significant coronary heart disease. Circulation 2009; 120:2207-2213.

36. Schaefer PW, Ozsunar Y, He J, et al. Assessing tissue viability with MR diffusion and perfusion imaging. AJNR Am J Neuroradiol 2003; 24:436443.

37. Reimer P, Saini S, Kwong KK, Cohen MS, Weissleder R, Brady TJ. Dynamic gadolinium-enhanced echo-planar MR imaging of the liver: effect of pulse sequence and dose on enhancement. J Magn Reson Imaging 1994; 4:331-335.

38. Ichikawa T, Arbab AS, Araki T, et al. Perfusion MR imaging with a superparamagnetic iron oxide using $\mathrm{T}_{2}$-weighted and susceptibility-sensitive echoplanar sequences: evaluation of tumor vascularity in hepatocellular carcinoma. AJR Am J Roentgenol 1999; 173:207-213.

39. Annet L, Materne R, Danse E, Jamart J, Horsmans Y, Van Beers BE. Hepatic flow parameters measured with MR imaging and Doppler US: correlations with degree of cirrhosis and portal hypertension. Radiology 2003; 229:409-414.

40. Breedis C, Young G. The blood supply of neoplasms in the liver. Am J Pathol 1954; 30:969977.

41. Sahani DV, Holalkere NS, Mueller PR, Zhu AX. Advanced hepatocellular carcinoma: CT perfusion of liver and tumor tissue--initial experience. Radiology 2007; 243:736-743.

42. Hagiwara M, Rusinek H, Lee VS, et al. Advanced liver fibrosis: diagnosis with 3D whole-liver perfusion MR imaging--initial experience. Radiology 2008; 246:926-934.

43. Huppertz A, Balzer T, Blakeborough A, et al. European EOB Study Group. Improved detection of focal liver lesions at MR imaging: multicenter comparison of gadoxetic acid-enhanced MR images with intraoperative findings. Radiology 2004; 230: 266-275.

44. Watanabe H, Kanematsu M, Goshima S, et al. Staging hepatic fibrosis: comparison of gadoxetate disodium-enhanced and diffusion-weighted MR imaging--preliminary observations. Radiology 2011; 259:142-150.

45. Keating GM, Santoro A. Sorafenib: a review of its use in advanced hepatocellular carcinoma. Drugs 2009; 69:223-240.

46. Schraml C, Schwenzer NF, Martirosian P, et al. Diffusion-weighted MRI of advanced hepatocellular carcinoma during sorafenib treatment: initial results. AJR Am J Roentgenol 2009; 193:W301W307.

47. Lewin M, Fartoux L, Vignaud A, Arrivé L, Menu $\mathrm{Y}$, Rosmorduc $\mathrm{O}$. The diffusion-weighted imaging perfusion fraction $f$ is a potential marker of sorafenib treatment in advanced hepatocellular carcinoma: a pilot study. Eur Radiol 2011; 21:281290.

48. Wu EX, Cheung MM. MR diffusion kurtosis imaging for neural tissue characterization. NMR Biomed 2010; 23:836-848.

49. Schmitt B, Zamecnik P, Zaiss M, et al. A new contrast in MR mammography by means of chemical exchange saturation transfer (CEST) imaging at 3 Tesla: preliminary results. Rofo 2011 ; 183:10301036. 